\title{
A systematic review of social and environmental factors and their implications for indoor cat welfare
}

\author{
Rachel Foreman-Worsley*, Mark J Farnworth \\ School of Animal, Rural and Environmental Sciences, Nottingham Trent University, Brackenhurst Campus, Nottinghamshire, NG25 OQF, UK
}

\section{A R T I C L E I N F O}

\section{Keywords:}

Cat

Feline

Indoor

Welfare

Behaviour

Enrichment

\begin{abstract}
A B S T R A C T
Cats are one of the world's most populous companion animals, yet little is known about how the home environment is adapted relative to their needs. Outdoor access is thought to be beneficial for both the physical and mental wellbeing of cats, yet as urbanisation increases, reducing owner access to outdoor spaces, an increasing number of cats are kept strictly indoors. The impact of an indoor lifestyle on feline behaviour and welfare is little explored and poorly understood. This study used a systematic review to assess scientifically validated knowledge concerning social and physical environments and their implications for indoor cats. A total of 61 papers were analysed. Only $n=21$ papers directly addressed at-home indoor scenarios with the remainder consisting of shelter/cattery $(n=27)$ or laboratory $(n=16)$ (some papers explored multiple environments). Across studies there was little evidence of rigour or systematically controlled approaches. Methods frequently used were catstress-scores (CSS) and ethograms, neither of which were consistently standardised, substantially reducing the ability to compare findings among studies. Numerous studies explored similar variables (i.e. provision of hiding space $(\mathrm{n}=9)$ ) yielding little additional knowledge. Measures of welfare and behaviour were often assessed using single parameters in controlled environments. Although this may be useful and applicable to cat experiences within shelters, catteries and laboratories, the findings do not necessarily translate to dynamic and variable household environments. Major findings include the benefits of enrichment such as hiding boxes and vertical resting spaces, as often recommended by veterinarians and feline charities. However, other advice provided, such as the provision of feeding enrichment for psychological welfare, although not necessarily disputed, appears to be scientifically untested. Additionally, despite the social environment being likely to have a substantial effect on cat welfare, it is particularly under-studied in the home, especially in terms of its complexity (e.g. presence of young children or dogs). Overall, the review identified substantial gaps relative to cat experiences and welfare in multifactorial home environments. Understanding the impact of indoor lifestyles and promoting mechanisms to minimise any negative impacts whilst promoting positive ones, remains an important, yet underexplored, area of research.
\end{abstract}

\section{Introduction}

In 2017 there were an estimated 10.3 million owned cats in the United Kingdom (UK) (PDSA, 2017) and 94.2 million owned cats in the United States of America (USA) (APPA, 2018). Housing practices for domestic cats (Felis catus) vary. Within Europe, cats are typically afforded an indoor/outdoor lifestyle whilst cats in the USA are more routinely kept indoors. Reasons such as fear of road traffic accidents, theft or attack by humans or animals play a role in the decision to keep cats as indoor-only (McLeod et al., 2015; Toukhsati et al., 2012). Increasingly, urbanisation is reducing owner access to outdoor spaces and so the number of indoor cats is expected to increase. Additionally, concern over the impact of cat depredation on wildlife is growing, especially in places where cats represent an introduced predator. Partial or complete indoor housing is seen as one mechanism to reduce the impact of cats on wildlife (Linklater et al., 2019). Suburbs in Australia have imposed a 'cat curfew', restricting cats to indoors during designated times, whilst other suburbs prohibit cats from being let outside entirely (Australian Capital Territory Government, 2018). In New Zealand, Marlborough District Council requires a multiple cat licence to be sought by those wishing to own more than four cats, to limit the cat population in areas where birds may be at risk (Marlborough District Council, 2019).

Typically, outdoor access has been accepted as beneficial for both the physical and mental wellbeing of cats (International Cat Care, 2019a), and little is currently known about how well cats adapt to an

\footnotetext{
* Corresponding author.

E-mail address: rae.foreman@ntu.ac.uk (R. Foreman-Worsley).
} 
indoor lifestyle. Compared to species such as dogs, cats have a relatively unique evolutionary history, with less intensive selection for domesticated traits (Driscoll et al., 2007). Consequently, cats exhibit behavioural characteristics and needs very closely aligned to their ancestors, including the drive to hunt, preference for a solitary lifestyle, territorial behaviour, crepuscular activity and a desire to bury faeces and urine (Bradshaw, 2018). As with all sentient species, poor welfare and problem behaviours may arise if behavioural needs are not met (Broom, 1986). In cats, common problem behaviours include inappropriate elimination, spraying, excessive vocalisation, scratching furniture and aggression towards owners, strangers or other animals (Heidenberger, 1997). These problems are reported to be more prolific in indoor cats compared to indoor/outdoor cats (Amat et al., 2009; Sandøe et al., 2017; Schubnel and Arpaillange, 2008). Some problem behaviours are found in almost twice the number of indoor cats compared to outdoor cats. For example, both Sandøe et al. (2017) and Schubnel and Arpaillange (2008) report significantly higher levels of house soiling or inappropriate elimination in indoor cats compared to outdoor cats, at the levels of $18.2 \%$ and $34 \%$ of indoor cats vs $9.8 \%$ and $16 \%$ of outdoor cats respectively. There may be an increase in cat-owner conflict if the behaviours are prolonged or particularly undesirable, potentially leading to abandonment or relinquishment. In the UK, it has been estimated that around $33 \%$ of cats relinquished to shelters were brought in due to behavioural problems (Casey et al., 2009).

Understanding which environmental aspects of a home may provide a positive experience for a cat is important to ensure good welfare. Cat welfare charities recommend provisions of food and water bowls, litter trays and scratching posts to meet basic needs. Additional recommended enrichment often alludes to hiding spots, vertical vantage spots and toys which can emulate hunting (Herron and Buffington, 2010; International Cat Care, 2019b). Yet environments are complex and multivariate, both physically and socially, thus the provision of structures and objects may only play a small role in providing for positive welfare. How cats perceive their daily interactions with people, conspecifics or heterospecifics such as dogs, and how this alters their behaviour, could be as important as the provision of physical items. Cats living in groups have been shown to occupy smaller ranges within a home (Bernstein and Strack, 1996), which may limit access to space and resources provided. Welfare may also be impacted, as when owners of both a cat and dog were surveyed, it was found that $20.5 \%$ of cats cohabiting with a dog were reported as uncomfortable in the dog's presence on a weekly basis (Thomson et al., 2018). Understanding the impact of each variable and their complex interactions is necessary to ascertain the extent to which indoor and outdoor environments can result in positive welfare for cats. Not only can an appropriate environment enhance their quality of life, but it can also impact upon the human caregiver. In dogs, it has been seen that reducing problem behaviours strengthens animal-owner bonds through increasing owner satisfaction with their animal (Van Herwijnen et al., 2018). In turn, strong animal-owner bonds provide benefits in terms of companionship and decreases the chances of animal relinquishment (Patronek et al., 1996).

Homes are not the only human mediated indoor environment in which cats reside. Annually, shelters in the UK and USA, see an estimated 150,000 (Clark et al., 2012; Stavisky et al., 2012) and 3.2 million (ASPCA, 2019) cats respectively. Cats are also routinely kept in research laboratories for studies of pet products and medical testing. In these spaces, cats are typically kept in smaller, confined spaces with reduced complexity, they may also have less human interaction and have a socially unstable environment. Individuals may be subject to changing caregivers and periods of intense interactions such as veterinary examinations or surgeries, before paradoxically facing sedentary lifestyles with little variation or stimulation. Many studies aim to provide knowledge to enhance welfare in these environments through correct care provision and appropriate enrichment. For example, the exploration of clicker training as a form of social enrichment and method of alleviating stress (Kogan et al., 2017), investigation into whether cats infected with Feline Immunodeficiency Virus (FIV) have different enrichment preferences to non-infected cats (Kennedy et al., 2018) or quantifying any effect of pheromones on stress levels (Chadwin et al., 2017). Although these environments diverge substantially from the typical home of a companion cat, they may be able to provide valuable insight as to how permanent indoor housing may affect cats.

This study uses a systematic review to collate and assess current scientific knowledge concerning the impact that the environment has on indoor-housed cats. By doing so it aims to elucidate what is currently known about those effects on the behaviour and welfare of cats. In addition, areas which require further exploration will be identified.

\section{Methodology}

\subsection{Scope of the literature}

For the purposes of this review, environment was taken to mean both the physical and social environments. For the physical environment (objective one) papers were deemed to be relevant if the welfare or behaviour of cats was being studied in any indoor capacity i.e. a home, laboratory, cattery or rescue centre. Research with an outdoor area was included if that area was enclosed, as were studies where cats were confined for the full duration of the research. Papers which utilised indoor/outdoor cats as well as indoor cats were included providing specific results pertaining to indoor only cats were discussed.

Regarding the social environment (objective two), research was included if it related to the interaction between cats and any form of caregiver (e.g. owner, shelter staff or researcher), conspecifics, or other species with whom they shared their indoor dwelling. Literature based on subjective report (i.e. those pertaining to studies addressing the human-animal bond and pet-owner attachment) were not included in the analysis, although they were retained for discussion.

\subsection{Searches}

The online databases Scopus (https://www.scopus.com/), Web of Science (WoS) (https://www.webofknowledge.com) and Google Scholar (GS) (https://scholar.google.co.uk/) were used to conduct the searches. These databases were chosen due to the quantity and availability of relevant journals and the breadth of literature covered. Boolean search phrases (see below) were used to retrieve the relevant literature. These searches were optimised through repeat iterations of the Boolean phrases until the literature returned was maximised.

Boolean phrase for objective one: (cat OR feli*) AND (indoor* OR shelter* OR pen* OR adopt* OR rescue* OR house* OR home*) AND (welfare OR behav* OR enrich* OR stress*)

Boolean phrase for objective two: (cat-owner OR owner-cat OR staff-cat OR cat-staff OR human-cat OR cat-human OR cat-caregiver OR caregiver-cat OR carer-cat OR cat-carer) AND (interact* OR behav* OR social* OR relat*)

Literature was exported into Mendeley for sorting. Due to the number of short phrases used within the search terms, substantial numbers of papers were returned. Returns were sorted by relevance. Using Scopus, the first 2000 citations were taken. From Web of Science, all citations were taken. From Google Scholar the first 350 citations were taken and the subsequent 200 citations (i.e. 351-550) checked for relevance. More details of the search items returned can be found in the results section, 3.2 .

\subsection{Literature filtering}

Literature retained was filtered using a step-wise process for both objectives. Only primary data were retained for analysis, whilst advisory material on enrichment and educational material on feline 
Table 1

Specific data extracted from each document classed as relevant to the systematic review.

\begin{tabular}{ll}
\hline Data categories & Variables \\
\hline Study characteristics & Year of publication, study origin, place of publication \\
Methodological characteristics & N number of participants, controls used, variables studied, measurement type, study length, limitations \\
Environmental characteristics & Social or physical environment, experimental setting, single or group housing \\
Cat characteristics & Age of cats, neuter status, sex \\
\hline
\end{tabular}

behaviour were retained for discussion only. Additionally, any papers in which an indoor environment was considered a minor or contributing variable, without being a substantial focus, were not included and likewise retained for discussion. Papers included were restricted to those published in English.

The process of refining the literature was as follows. Firstly, duplicates of citations retrieved by multiple databases were merged within the Mendeley desktop program. Secondly, titles were used to determine if the work was related to the domestic cat; if not it was removed. Titles were used again to remove citations not obviously relevant to indoor or social environments. Next, abstracts were assessed. Finally, the full-text was read to ensure the record was relevant to the research objectives.

\subsection{Data extraction}

Once the relevant material was identified, pertinent data from each paper were extracted and inputted into Microsoft Excel. Data extracted can be found in Table 1, in addition to the citation. For a summary of each paper's major findings, see suppl. 1. Papers were also categorised into groups based on the relevant research objective, with some papers being relevant to both objectives. More details on the categorisation of papers for each research objective can be found below.

\subsubsection{Environment}

Three major research environments: house, laboratory and shelter/ cattery, were identified. House environments were the homes of owners in which the cat permanently resided. Laboratory studies were those conducted within a dedicated research facility. For the latter, cats were typically born in the facility and received daily care from a research team, although some studies $(n=5)$ used cats taken from shelters or homes to a research facility for the duration of the experiment and subsequently returned. Shelters and catteries were grouped for analysis due to environmental similarities where cats were confined to pens or rooms. Feeding, cleaning and grooming were routinely provided by various carers and the environment could contain sights, sounds and smells of unknown conspecifics.

\section{Results}

The results returned for the search terms in each of the databases are detailed in Fig. 1. In total, 61 papers were retained for analysis; 33 papers arising from search objective one and 28 papers from search objective two. Of the 61 papers, the foci were: social environment $\mathrm{n}=7$, physical environment $\mathrm{n}=11$ and both social and physical environments $n=43$. A summary of each of the papers can be found in the supplementary material.

\subsection{Analysis of data}

\subsubsection{Date of publication}

Of the data retained, $\mathrm{n}=16$ papers were published between 1991-2000, $\mathrm{n}=11$ between 2001-2010, and $\mathrm{n}=34$ between 20112018.

\subsubsection{Research environments}

Of the studies that utilised one research environment, the most frequent was a shelter/cattery $(n=23)$, then the home environment $(n=18)$ and then laboratory $(n=16)$. Four studies compared results from multiple environments, with $\mathrm{n}=2$ comparing shelters and houses, $\mathrm{n}=1$ a cattery and house and $\mathrm{n}=1$ a shelter and cattery.

\subsubsection{Social interactions}

Of the 50 papers that considered behaviour and welfare based on social interactions, $n=24$ explored cat-cat and $n=15$ cat-human and $\mathrm{n}=2$ cat-dog. Nine papers considered multiple social relationships between humans, cats and/or dogs. The three papers covering cat, dog and human interaction used survey methodology to consider the overall home environment, which included cat-owner interaction and, briefly, the presence of other cats or dogs (Adamelli et al., 2005; Heidenberger, 1997; Shyan-Norwalt, 2005).

\subsubsection{Measures of behaviour and welfare}

Methodologies employed to assess behaviour and welfare predominantly fell into distinct groups, the majority of which are discussed below. More than one method was utilised by $n=31$ papers.

3.1.4.1. Observations. Behavioural observations were used in $n=47$ studies. Observations were collected through video recordings $(n=18)$ and/or the presence of an observer $(n=30)$. Observations were sampled continuously $(n=29)$ and/or via scan sampling $(n=22)$. The data recorded from observations were most commonly explored using ethograms $(n=32)$ to record frequency and/or duration of behaviours. Data were also used to measure cat-stress-score (CSS) $(\mathrm{n}=13)$ (discussed further below).

3.1.4.2. Survey. Different forms of surveys or questionnaires were used for $\mathrm{n}=13$ papers. These were often directed at owners and assessed physical environmental composites (Strickler and Shull, 2014) or social interaction (Rochlitz et al., 1998b). Personality was explored by Ramos et al. (2013) who asked owners to assess their cat's personality.

3.1.4.3. Physiological measures. These were assessed in $n=8$ papers. One paper used two physiological measures and so nine methodologies were used in total. Measurements such as cortisol levels, immune function and creatinine:cortisol levels were taken from blood $(n=2)$, urine $(n=4)$ and faeces $(n=3)$.

\subsubsection{Major findings}

Many studies focused on similar areas. A summary of the major findings across multiple studies can be found below, whilst a brief summary of each study can be found in the supplementary material. The major findings are discussed further in 3.1.6, the limitations section, and Table 3.

\subsubsection{Limitations}

As detailed in Table 3, numerous limitations were identified, with the major two being small sample sizes and inconsistent methodologies across repetition of study objectives.

Limitations were decided through consideration of good experimental design, such as the use of large sample sizes and methodological consistency. As detailed by Taborsky (2010), sample sizes for behavioural studies do not appear to be standardised, with chosen sample 


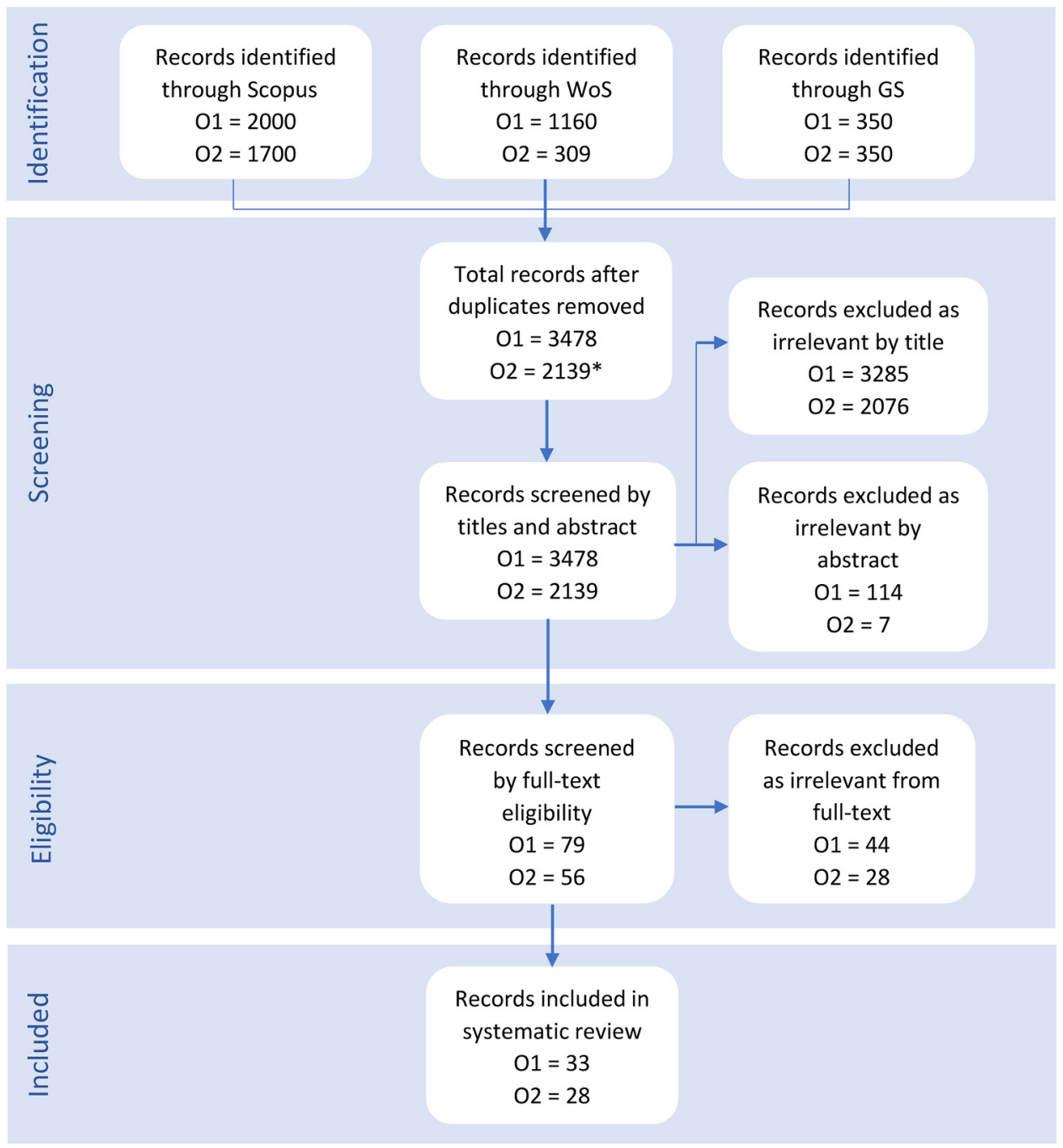

Fig. 1. Flow chart depicting the number of citations obtained from each search engine for objective one: physical environment (O1) and objective two: social environment (O2), and the records removed at each step based on the eligibility criteria. The flow chart has been adapted from Moher et al. (2009). When duplicate records from objective two were removed these included duplicates of records found using the objective one search terms.

sizes often arising from the number of subject available, limited resources, time constraints or convenience, rather than being optimised. Whilst optimal sample sizes vary depending on the analysis being completed, where $\mathrm{N}=<25$, studies were highlighted as having a potential issue with small samples sizes. $\mathrm{N}=1$ studies were highlighted separately, due to the more preliminary status of the findings.

Other limitations were experimental design details which may have inadvertently impacted results by introducing variation and reducing comparability between individuals or groups in the study. For example, Broadley et al. (2014) utilised cats who had entered the shelter within the previous 7 days. Results of behavioural observations can be expected to differ between a cat residing in a new environment, in this case a shelter, for 1 day when compared to 7 days. Whilst time on site before observation was considered as a variable, as each individual is likely to acclimate at a different rate from a different starting level of stress, this is still a limitation of the experiment.

Studies with sample biases are limited as the results may not be applicable to all cats. For example, Strickler and Shull (2014) distributed surveys to attendees of veterinary hospitals to assess toys, activities and problem behaviours, and Feuerstein and Terkel (2008) recruited cat owners from notices placed around a university. Convenience sampling may inadvertently select owners who are more conscientious or provide better care for their cats, as they are willing to attend a veterinary centre or more keen to participate in research.
Whilst demographic, representative samples are not required, it must be considered that not all cats are living in such environments.

Whilst some limitations listed in the table are not necessarily true limitations, they can be defined as problems which are limiting this field of work e.g. repetition of box studies.

3.1.6.1. Small sample sizes. These were identified in $\mathrm{n}=32$ papers, with an additional $\mathrm{n}=9$ papers being effectively $\mathrm{N}=1$ studies. Studies were classed as having a small sample size if they had $n=<25$ participants in any given experiment. The smallest samples were $n=7$ (Rochlitz et al., 1998a), $n=13$ ( $n=9$ and $n=4$ in the experimental and comparison groups respectively; Soennichsen and Chamove, 2002) and $\mathrm{n}=16$ ( $\mathrm{n}=8$ and $\mathrm{n}=8$ in the experimental and comparison groups; Carlstead et al., 1993) Some papers started with larger samples, however, comparing results between experimental groups substantially diminished sample sizes. For example, Ramos et al. (2012) used $n=30$ subjects, $n=14$ housed singly and $n=16$ housed in groups, reducing independent samples to $\mathrm{n}=14$ individuals and $\mathrm{n}=6$ groups. Desforges et al. (2016) used $\mathrm{n}=48$ individual cats, but only $\mathrm{n}=4$ groups. Bradshaw and Hall (1999), who were studying pairs, used $n=50$ individuals, therefore $\mathrm{n}=25$ pairs, and compared results between related and unrelated pairs, taking the group sizes down to $n=14$ and $\mathrm{n}=11$ respectively. Studies with the smallest samples were frequently those studying large group behaviour. Many of these 
studies ( $\mathrm{n}=9$ ) were found to be $\mathrm{n}=1$ studies (as detailed in Table 3 ), as only one group population with multiple members was utilised per study.

3.1.6.2. Inconsistent methodology. Similar methodologies were used across numerous studies, namely CSS, ethograms and humanapproach-tests (HATs), however, the methods were often inconsistent or modified reducing the comparability across studies.

3.1.6.3. Cat-Stress-Score. Cat-stress-score (CSS) as devised by Kessler and Turner (1997), is utilised in $\mathrm{n}=13$ papers. CSS is often used to monitor stress over time, either in response to a new environment or to compare stress between treatment groups and a control group, when provided with items such as a hiding box (Vinke et al., 2014) or pheromones (Chadwin et al., 2017). The original methodology for measuring CSS averaged two CSS recorded within 15 min of each other. Generally, most studies used the mean of at least two scores per recording, although Broadley et al. (2014) and Chadwin et al. (2017), utilised only one. Vinke et al. (2014) used four CSS measurements taken within $20 \mathrm{~min}$ to form an average. McCobb et al. (2005) recorded two scores, but after an interval of 'at least 15 min', suggesting the interval times may have varied.

3.1.6.4. Ethogram. Behavioural assessment using an ethogram was the most frequently employed methodology $(n=30)$, however, there was little standardisation between studies. Some studies used comprehensive ethograms to record a wide scope of behaviours (Podberscek et al., 1991) whilst others had a strict focus, such as Snowdon et al. (2015) who were recording behavioural responses to music. Several papers studying cat-cat interactions categorised behaviours as affiliative or agonistic, yet again, these behaviours were not standardised (Barry and Crowell-Davis, 1999; Dantas-Divers et al., 2011). Typically, a single observer coded the ethogram, either whilst observing in real-time or from a video after the event, although not all papers detailed how many observers completed the ethogram (Feuerstein and Terkel, 2008). Training on the use of ethograms was not detailed. Where multiple observers were used and interobserver reliability identified, high levels of agreement were reported. Kogan et al. (2017) detailed a correlation of 0.990 and Snowdon et al. (2015) a concordance of $94.5 \%$.

\section{Discussion}

Research into the behaviour and welfare of indoor cats is a growing area of interest, with 61 papers identified since 1991, and more papers published in the last decade than the two previous decades combined. Despite this, the current breadth of knowledge remains small when considering the complexity of environmental variables that may affect the behaviour and welfare of individual cats.

\subsection{Major findings}

\subsubsection{Cat-cat literature}

Cat-cat interaction was frequently studied in group housing. For cats who have not chosen to cohabit, as those within feral colonies may do out of necessity, understanding group interactions with conspecifics is important to minimise conflict and maximise welfare.

When providing enrichment, it is important that enough enrichment is provided to ensure all cats can access it. Large enrichment items such a cat trees or benches were shown to be utilised by multiple cats at once (Loberg and Lundmark, 2016). Yet with smaller items, it was found that within groups certain individuals more frequently commandeered the use of enrichment items (Bernstein and Strack, 1996; Damasceno and Genaro, 2014; Loberg and Lundmark, 2016). Once additional resources were added, these were utilised by other individuals (Bernstein and Strack, 1996; Damasceno and Genaro, 2014). This indicates it was likely that group social structure and dynamics, as opposed to lack of interest, modified enrichment use. Typically, feline behavioural experts recommend providing one of each resource type (e.g. litter tray, water bowl) per cat, plus one spare, to reduce conflict and improve welfare, yet no literature within this review was found to investigate this recommendation. As of now, it appears that this claim is untested, although it may still be beneficial.

Cats appear to have preferred areas within their environment, which they frequent more often or in which they spend significantly more time (Barry and Crowell-David, 1999; Bernstein and Strack, 1996). Although cats have been shown to time-share spaces and resources (Bernstein and Strack, 1996), ensuring that cohabiting cats can access a variety of resource-rich areas may promote good welfare by reducing conflict over home ranges. This advice is often provided by feline behavioural experts, although whether resource dispersion is beneficial in reducing conflict or improving welfare is untested as of yet. In general, an increase in space, or decrease in population density, may be beneficial to welfare. For example, Loberg and Lundmark (2016) found increased space improved play behaviour, a commonly accepted indicator of positive welfare.

Aggression was frequently studied. Ethograms included subtle displays of aggression such as piloerection, ear turning or flattening and staring, alongside more overt displays such as pouncing, mounting, swatting or biting (Barry and Crowell-Davis, 1999; Dantas-Divers et al., 2011; Lichtsteiner and Turner, 2008), although not all ethograms were standardised. Observations conducted across laboratory, shelter and home environments and of varying group sizes with differing sex ratios, generally all found low rates of conflict (van den Bos et al, 1994, 1998b; Bernstein and Strack, 1996; Dantas-Divers et al., 2011). Pairs of cats that have lived together longer in homes appear to have reduced rates of aggression than those cohabiting for shorter periods (Barry and Crowell-Davis, 1999). This may be due to the dynamics of the social dyads being more stable, as hierarchies and home ranges are well established. Cohabiting cats with high levels of aggression may also face relinquishment, removing them from the population of animals being studied.

Whilst low conflict may be considered more neutral than a positive marker of a relationship, several indicators of affiliative relationships were established. Related cats kept in a shelter environment were found to have more affiliative behaviours than unrelated pairs (Bradshaw and Hall, 1999), whilst sex had no impact on affiliation in related or unrelated pairs. Barry and Crowell-Davis (1999) additionally found that sex had no impact on affiliative behaviours or aggression in pairs living together, although it did appear to influence proximity, with male/male pairs spending more time near one another.

\subsubsection{Cat-owner literature}

A major feature in the environment of cats in homes is the owners or family with whom the cat resides. As the primary caregiver, an owner's actions or personality can impact on the welfare of their cat (Finka et al., 2019). In the indoor environment especially, cats only have access to items that the owners have provided, intentionally or otherwise. This may be problematic as solely indoor cats do not have the opportunity to seek out provisions or items to meet their needs. Adamelli et al. (2005) found owner gender, education, previous experience and number of friends were major factors influencing the care provided for cats and thus the cat's quality of life.

In addition to physical provisions and resources, there is a social relationship between the cat and the owner. This dyad has been suggested as more important than cat-cat dyads, or the physical environment in impacting welfare (Adamelli et al., 2005; Lichtsteiner and Turner, 2008; Ramos et al., 2012). The cat-owner relationship seems in many instances to be beneficial to the cat although this is not necessarily always the case (Finka et al., 2019). People who spent several hours throughout the day with their cats reported fewer problem behaviours (Heidenberger, 1997). A study on owner-cat separation 
showed that after spending $4 \mathrm{~h}$ apart from one another, cats purred more at reunion than they did after 30 min (Eriksson et al., 2017). When a 3-month separation period was studied for cats in quarantine, $\sim 0 \%$ of owners reported their cat to be more attached to them 2-weeks and 3-months post-release than before quarantine, and 3-months after release they reported their cats spent more time with them than before quarantine (Rochlitz et al., 1998b). These results post-quarantine may potentially indicate low levels of separation anxiety, an interesting area for further study.

\subsubsection{Enrichment}

There is robust evidence that hiding enrichment is utilised by cats when given the opportunity and that it positively impacts welfare. Within a novel environment, such as entry into a shelter or cattery, hiding provisions reduce behavioural and physiological stress markers. Cats displaying evidence of stress utilise hiding opportunities more (Ottway and Hawkins, 2003; Rehnberg et al., 2015; Stella et al., 2014, 2017). Cats with hiding enrichment displayed more rapidly declining CSS than those without (Kry and Casey, 2007; Vinke et al., 2014). Cats with hiding enrichment were observed to spend more time inactive compared to control groups (Kry and Casey, 2007; de Oliveira et al., 2015), indicating they were more comfortable within their own environment.

Hiding motivation was explored by comparing comfortable resting spots to hiding areas by Vinke et al. (2014). When provided, hiding boxes were the most utilised area, leading the authors to conclude that hiding boxes are not just comfortable, but have a 'main concealing function'. Further evidence that motivation is concealment over comfort can be seen within other studies, where cats lacking hiding enrichment exhibited behaviours that researchers interpreted as trying to hide. These behaviours were described as 'resting behind the litter pan' by Carlstead et al (1993), 'crouching behind their beds' by Kry and Casey (2007) and spending time 'behind their litter box' by Vinke et al. (2014).

\subsection{Limitations}

\subsubsection{Inconsistent methodologies}

Despite many studies utilising similar methodologies, inconsistencies diminish the ability to compare results between papers thus making a statistical meta-analysis of previous data not possible. Cat Stress Score is one of the most utilised methods within the reviewed papers $(n=13)$. It has benefits in that it is quick, can be performed by anyone with little training, and is non-invasive, meaning it is a valuable tool for researchers and those interacting with cats daily. However, its application did not always conform to the methodology devised by Kessler and Turner (1997).

Despite being easy to use, it is uncertain how frequently CSS was being applied in a correct or valid way. During its development Kessler and Turner (1997) detailed that interobserver reliability is high, at 0.9, when utilised by two trained observers, yet in those with less training this reliability drops to 0.75 . The papers in this review scarcely detail the training undergone in CSS prior to use, so it is not certain that observers across different studies are consistent with their scoring. Additionally, most papers utilised one observer which may reduce reliability when compared with average scores from multiple observers. Loberg and Lundmark (2016) did utilise multiple observers, however scores were not recorded simultaneously and averaged, meaning multiple observers may reduce consistency between scores. Whilst it was detailed that observers practiced together to ensure interobserver reliability, no quantified score was provided. Broadley et al (2014) also used multiple observers, and only reported an interobserver correlation of 0.64 between the researcher and an experienced observer, further highlighting issues with consistency across the application of CSS.

Where CSS was utilised, the behaviours used to score stress levels between 1 and 7 were adhered to by all papers, although Vinke et al.
(2014) omitted vocalisation. Variation in the methodology of measuring CSS was found within repeated measurements. Repetition improves reliability, however some studies omitted repetition and averages (Broadley et al., 2014; Chadwin et al., 2017), whilst others extended the original average of two measurements to an average of four measurements (Vinke et al., 2014), and there was often variation in the time between measurements, from 5 min per observation, to 'at least' 15 min between observations (McCobb et al., 2005).

The CSS measurements also varied relative to the time of day they were recorded. Broadley et al. (2014) measured CSS between 12:00 h $15: 00 \mathrm{~h}$, as they suggest scores may vary throughout the day as cortisol levels do. McCobb et al. (2005) found CSS was significantly higher in the morning, although Loberg et al. (2016) did not find this was the case. Whilst variations in McCobb et al. (2005) may be due to environmental factors, controlling for potential variables where possible, such as time of day, could increase reliability when comparing studies.

Overall, despite the inconsistent application of CSS, papers with similar foci, for example CSS over time, do broadly agree with one another. Variations in findings may be attributable to methodological differences or other confounding variables.

\subsubsection{Small sample sizes}

Several papers derived findings from $\mathrm{N}=1$ studies. This lack of independent measures within experimental designs reduces, or removes, the ability to extrapolate findings to the wider population. Typically, $\mathrm{N}=1$ studies were group studies investigating social behaviour. It is perhaps due to the scale of these studies, including the length and quantity of data to be processed, as well as access to subjects, that only one group was utilised. Whilst it is possible to compare results between similar $\mathrm{N}=1$ studies, differences such as the number of cats in the colony, the size of the environment, the enrichment present or the presence of humans, means conclusions are reduced to generalised statements, and more specific nuance amongst results remain underinvestigated.

\subsubsection{Repetitive studies}

Whilst it is important that replication establishes findings are valid, $15 \%(n=9 / 61)$ of all studies had a large, intentional focus on the impact of a hiding box on behaviour and welfare. This can be considered excessive. This is especially so when all studies concur that boxes are well utilised by cats and beneficial to their behaviour and welfare. Additional studies included hiding boxes as a provision of enrichment whilst investigating other variables. Whilst hiding behaviour may have been included on ethograms, this was not a substantial focus and thus these studies were not included in the count. Given the many variables within a cat's environment, there remains substantial opportunity to explore other environmental variables that may influence behaviour and improve welfare.

\subsubsection{Application to home environment}

Whilst most indoor cats globally are found within homes, most of the studies were completed in a shelter, cattery or laboratory. Advice and guidance documents providing recommendations for ensuring good welfare in the home are therefore likely to use results obtained in environments other than the home. It must be considered that these results may not be transferrable between environments. Indeed, inconsistencies in results between similar studies may be down to different environments and consequently, guidelines for enrichment found in the non-peer reviewed literature pertaining to the welfare of indoor cats, remain to be scientifically validated.

Hiding enrichment, for example, was primarily investigated in shelters, catteries and laboratories. Studies often focused on adaptation to a new, relatively barren, environment, which may be less relevant to cats living in homes where complexity is substantially greater. Bernstein and Strack (1996) provided boxes within a group home environment to explore social structure through observation. Here, boxes 
were found to be utilised for several hours a day when introduced, but a novelty effect meant that after 5 days usage diminished. With the introduction of a new box the interest once again increased. Comparing studies within shelters with those in the home environment suggests that boxes may be utilised differently by cats in a stable environment versus those in a novel environment. Hiding enrichment could still be beneficial in the home for indoor cats but may be superseded by incidental and permanent hiding spaces (e.g. behind or underneath other structures).

Feeding enrichment is additionally promoted as beneficial as it increases activity and alleviates boredom therefore improving welfare (Ellis et al., 2013; Stella et al., 2017), yet these claims are not wholly demonstrated in the literature. Whilst Dantas-Divers et al. (2011) and Damasceno and Genaro (2014) both found that feeding enrichment was interacted with by many individuals within a group when provided in a shelter, Naik et al. (2018) found that, compared to standard bowl feeding, exclusively using puzzle feeders did not increase activity levels in cats in the home. No welfare measurements of cats provided with enrichment were taken and so, whilst puzzle feeders were interacted with, it cannot be said whether this significantly impacted upon welfare. These examples demonstrate the need for large-scale, in-home studies into behaviour and welfare to ensure that research is applicable to, and beneficial to, a large a population of cats as possible.

\subsection{Knowledge gaps}

\subsubsection{Long-term studies}

Many studies investigated the time taken for cats to adapt to a new environment and ways to mitigate the stress faced during this adjustment period. Overall, the consensus was that a 3-5 day period was required for CSS to fall to an acceptable level, (Table 2). Whilst this is important in scenarios where cats are being rehomed or boarded, it does not help us to understand long-term stress within complex environments. Chronic stress and the concomitant changes in cortisol levels may have a more detrimental effect on welfare than acute stress. Systemic cortisol elevation over a long period can cause metabolic changes and decreased immune function, increasing susceptibility to disease (Heimbürge, 2019). Whilst it is important to mitigate stress in all scenarios, home-life typically comprises the largest number of cats, and the majority of those cats' lifetimes. Therefore, increasing welfare in the home, and our understanding thereof, will have the greatest impact on cat welfare overall. Within this review, the only long-term studies focused on cats spending six months in quarantine, thus it is reasonable to state these results will not contribute towards understanding welfare in the home.

\subsubsection{Complex environments}

As is typical in experimental design, many studies focus on a single variable, for example, litter tray preference (Grigg et al., 2012), reaction to auditory stimulation (Snowdon et al., 2015) or effect of feeding enrichment (Damasceno et al., 2014). Studies with one variable help to ensure any differences between groups most likely result from the factor under investigation. Whilst exploring individual variables within the environment is important, it is also important to understand the complex interactions between the numerous variables in a given environment and how they can impact behaviour and welfare as a whole. The presence of one item in a study may be beneficial to behaviour or welfare in an otherwise stark environment, yet in practice, the benefits of the item may be inconsequential in a complex environment. Preference tests are a good way of determining the importance of objects to cats. Yet as preference tests tend to be short-term studies, there could be a potential novelty effect which diminishes over time and thus removes the long-term benefits of the object. Heidenberger (1997) and ShyanNorwalt (2005) utilised surveys to gain an insight into the different aspects of the home environments of respondents' cats. These surveys are useful in evaluating what recommended enrichment is being provided for cats, the interaction cats have with their environment and the social interaction cats experience. If these aspects could be linked to indicators of welfare, it may be possible to determine which factors have the greatest impact in the home. Strickler and Shull (2014) used owner surveys to link play behaviour and enrichment to aggressive or problematic elimination behaviours in the home. They identified several variables associated with increased problem behaviours. For example, owners who played with their cats for five minutes at a time reported fewer problem behaviours than owners who played for one minute at a time. Extending surveys to include a stronger emphasis on problem behaviours could substantially develop our understanding of the environmental factors that have the biggest influence over cat

Table 2

A summary of the major findings compiled from similar studies included in this review.

Box/hiding enrichment

CSS over time

Stress in single vs group housing
Provision of hiding enrichment was primarily associated with reduced CSS compared to a control group without hiding enrichment (Kry and Casey, 2007; Vinke et al., 2014), although Moore and Bain (2013) found no significant effect of hiding or toy enrichment on CSS. A change in behaviour was seen compared to control cats without enrichment, including increased active and inactive behaviours (de Oliveira et al., 2015) and an increase in relaxed behaviours and human approach behaviours (Kry and Casey, 2007).

Increased utilisation by cats experiencing more stress (Ottway and Hawkins, 2003; Rehnberg et al., 2015; Stella et al., 2014, 2017). Preference of hiding enrichment over other forms of enrichment (Ellis et al., 2017b).

CSS was found to change following entry into a new environment, such as a shelter or cattery. Longer length of stay correlated with decreased cat stress scores within the first week (Broadley et al., 2014; Kessler and Turner, 1997, 1999a; Kry and Casey, 2007; McCobb et al., 2005; Moore and Bain, 2013; Rehnberg et al., 2015; Vinke et al., 2014) and second week (Kessler and Turner, 1997; Kry and Casey, 2007; McCobb et al., 2005; Vinke et al., 2014), although one study found that at two weeks in an unenriched environment, CSS started to increase compared to after one week (Kry and Casey, 2007).

After significantly declining from day 1, CSS was found to stabilise between 3-5 days depending on conditions such as single or group housing, prior socialisation or enrichment (Broadley et al., 2014; Kessler and Turner, 1997, 1999a; Kry and Casey, 2007; McCobb et al., 2005; Moore and Bain, 2013; Vinke et al., 2014)

CSS decreased faster in cats with hiding enrichment than those without (Kry and Casey, 2007; Vinke et al., 2014), faster in cats housed in groups compared to singly (Kessler and Turner, 1997) and faster in those with more human interaction (Rehnberg et al., 2015).

Cats not socialised with people had a higher overall CSS compared to those socialised with people in both group and single housing and cats not socialised with cats had a higher overall CSS when housed in groups compared to when housed singly (Kessler and Turner, 1999a). Results on stress in single and group housing were inconclusive. For cats socialised with other cats several studies found no difference in stress indicators between single and group housing. Kessler and Turner (1997, 1999a) found no difference in CSS between cats in single or group housing. Single and group housing was found to have no impact on faecal glucocorticoid metabolites (GCM) across two studies (Ramos et al., 2012, 2013). Lichtsteiner and Turner (2008) found single or group housing had no influence on urinary cortisol level.

Other studies however did find a difference. Two studies found group housing was less stressful than single housing. Gourkow and Fraser (2006) found CSS was generally higher in single, unenriched cages compared to unenriched group or enriched group/single housing. Uetake et al. (2013) found urine cortisol-to-creatinine ratios were generally higher in single housed cats than group housed. Ottway and Hawkins (2003), however, found CSS was higher in group housing than single housing.

Cats not socialised with other cats displayed more stress in group housing than single housing (Kessler and Turner, 1999a). 
Table 3

Limitations of the relevant articles included in the systematic review.

Limitation
$\mathrm{N}<25$ (some or all parts of experiment)

$\mathrm{N}=1$ study

Modified cat stress score

Inconsistent ethogram

\begin{abstract}
Short study length effects
\end{abstract}

Study exploring pre-established Box/hiding enrichment

CSS over time

Stress in group vs single

housing

Varying acclimation time pre-trial between individuals

Observer present

Sample bias

Low interobserver reliability

\section{References}

Barry and Crowell-Davis, 1999; Bradshaw and Hall, 1999; Carlstead et al., 1993; Desforges et al., 2016; Ellis et al., 2015, 2017a; Eriksson et al., 2017; Fazio et al., 2017; Gouveia et al., 2011; Kennedy et al., 2018; Kessler and Turner, 1997, 1999b; Kry and Casey, 2007; Lichtsteiner and Turner, 2008; Loberg and Lundmark, 2016; McGlone et al., 2018; de Monte and le Pape, 1997; Moore and Bain, 2013; Naik et al., 2018; Ottway and Hawkins, 2003; Ramos et al., 2012, 2013; Rehnberg et al., 2015; Rochlitz et al., 1998a, 1998b; Soennichsen and Chamove, 2002; Stella et al., 2014, 2017; Uetake et al., 2013; Vinke et al., 2014; Vitale Shreve et al., 2017; Zhang et al., 2018

Bernstein and Strack, 1996; Damasceno and Genaro, 2014; Dantas-Divers et al., 2011; de Oliveira et al., 2015; Parker et al., 2017; Podberscek et al., 1991; van den Bos, 1998a, 1998b; van den Bos and de Cock Buning, 1994

Broadley et al., 2014; Chadwin et al., 2017; Gourkow and Fraser, 2006; Kessler and Turner, 1997, 1999a, 1999b; Kry and Casey, 2007; Loberg and Lundmark, 2016; McCobb et al., 2005; Moore and Bain, 2013; Ottway and Hawkins, 2003; Rehnberg et al., 2015; Vinke et al., 2014

Barry and Crowell-Davis, 1999; van den Bos, 1998a, 1998b; van den Bos and de Cock Buning, 1994; Bradshaw and Hall, 1999; Dantas-Divers et al., 2011; Desforges et al., 2016; Ellis and Wells, 2008; Ellis et al., 2015; Eriksson et al., 2017; Feuerstein and Terkel, 2008; Gouveia et al., 2011; Loberg and Lundmark, 2016; Mertens, 1991; McGlone et al., 2018; de Monte and le Pape, 1997; Moore and Bain, 2013; de Oliveira et al., 2015; Ottway and Hawkins, 2003; Podberscek et al., 1991; Rehnberg et al., 2015; Rochlitz et al., 1998a; Snowdon et al., 2015; Soennichsen and Chamove, 2002; Stella et al., 2014, 2017; Uetake et al., 2013; Vinke et al., 2014; Vitale Shreve et al., 2017; Wedl et al., 2011

Damasceno and Genaro, 2014; de Oliveira et al., 2015; Rehnberg et al., 2015; Stella et al., 2014, 2017

Ellis et al., 2017b; Kry and Casey, 2007; Moore and Bain, 2013; de Oliveira et al., 2015; Ottway and

Hawkins, 2003; Rehnberg et al., 2015; Stella et al., 2014, 2017; Vinke et al., 2014

Broadley et al., 2014; Gourkow and Fraser, 2006; Kessler and Turner, 1997, 1999a; Kry and Casey, 2007; McCobb et al., 2005; Moore and Bain, 2013; Rehnberg et al., 2015; Vinke et al., 2014

Kessler and Turner, 1997, 1999a; Gourkow and Fraser, 2006; Lichtsteiner and Turner, 2008; Ottway and Hawkins, 2003; Ramos et al., 2012, 2013; Uetake et al., 2013

Broadley et al., 2014; Kessler and Turner, 1999a; Kry and Casey, 2007; Ottway and Hawkins, 2003; Vinke et al., 2014; Vitale Shreve et al., 2017

Barry and Crowell-Davis, 1999; Bradshaw and Hall, 1999; Gourkow and Fraser, 2006; Mertens, 1991; Rehnberg et al., 2015; van den Bos, 1998a, 1998b; Wedl et al., 2011

Ellis et al., 2015; Feuerstein and Terkel, 2008; Heidenberger, 1997; Kessler and Turner, 1997, 1999a, 1999b; Ramos et al., 2012, 2013; Strickler and Shull, 2014; Shyan-Norwalt, 2005; Thomson et al., 2018 Broadley, et al., 2014; Kessler and Turner, 1997, 1999a, 1999b behaviour and welfare in the home.

\subsubsection{Cat-child relationships}

Several studies allude to the possibility that the impact of humans in the social environment has a greater effect on welfare and behaviour than that of conspecifics (Adamelli et al., 2005; Lichtsteiner and Turner, 2008; Ramos et al., 2012). Despite this, more studies considered cat-cat relationships $(n=33)$ than cat-human relationships $(n=24)$. Additionally, the studies in this review exploring cat-human relationships focus predominantly on adult relationships, and cat-child interactions remain largely unexplored. The cat-child relationship is briefly explored by Heidenberger (1997), who found cats in households with children displayed less problem or anxious behaviours, and Mertens (1991) who found cat-child dyads made more approach behaviours than cat-juvenile or cat-adult dyads. These findings were small components within larger studies and so it is difficult to conclusively identify how the presence of children may impact upon cats. It could be reasoned that children may be intimidating to cats of certain dispositions due to the potential for unpredictable movement and sound, loud noises or heavy handedness. A study by Hart et al. (2018), suggested cat-child relationships are perceived as less affectionate and more problematic than cat-adult relationships. Single cat relationships with children were found to be less affiliative than those in multi-cat households. This may be due to having more attention directed towards them if they are the only cat. For indoor cats especially, inability to escape the attention of children, or any human resident, may be a chronic stressor reducing overall welfare.

\subsubsection{Cat-dog relationships}

Little literature exists relative to cats and dogs who cohabit $(n=5)$. A recent survey (Murray et al., 2009) estimates that 7\% of UK households contain both a cat and a dog, suggesting this dynamic affects many cats. Feuerstein and Terkel (2008) and Thomson et al. (2018) both investigated the cat-dog relationship specifically, whilst two others briefly incorporated the cat-dog dyad into larger cat lifestyle surveys (Heidenberger, 1997; Shyan-Norwalt, 2005). As dogs were a minor variable within the latter research there is little established knowledge concerning the relationship between the species and its impact on behaviour and welfare. Both studies of cat-dog relationships relied heavily on owner-reported relationships and quantification of owner perception of interactions. Owner perceptions can be largely subjective, and there may be limited behavioural understanding of the interactions or differences in the interaction being reported, leading to incorrect interpretation and report. Whilst Feuerstein and Terkel (2008) used video recording to quantify frequency of interactions, this single study was limited to 45 subjects preventing overarching conclusions from being drawn around the intricacies of cat and dog relationships. It does however provide a good grounding for future investigation.

\subsubsection{Validity of CSS used in social situations}

CSS was widely used in social groups in addition to single cats. CSS was developed by Kessler and Turner (1997) as an adaption of the CatAssessment-Score laid out by McCune (1994). Kessler and Turner (1997) detail how the revised ethogram was applied to single, pair and group-housed cats during its development and is thus applicable to cats in any housing situation. Yet the ethogram does not contain any measures pertaining to social behaviour, such as allogrooming, sniffing or vocalisations. This may reduce its validity in social situations. As indicated by the authors during the development of CSS, it would be beneficial to validate the behaviours against physiological parameters. This would help to confirm whether behavioural stress indicators are the same for cats housed singly or in groups. For now, when utilising CSS measurements of cats housed in groups, it is worth considering that the results may be less valid for those housed singly. 


\section{Conclusion and recommendations}

The relationship between cats and complex home environments is of increasing importance due to the rising numbers of cats being kept indoors. The dearth of studies in the home indicates the requirement for more research. As many studies concerning hiding enrichment, cat-cat social behaviour and relationships, and group and single housing have been conducted in shelters, catteries and laboratories inference is difficult. Substantially more cats live in homes meaning that improving our understanding of the impact of the home environment is imperative to improving companion cat welfare. As access to the home environment presents methodological challenges it remains largely unexplored. This area may be filled most readily by feline behavioural consultants who already have regular access to assessing behaviour in this environment.

To optimise the impact of research, focus should be placed on the gaps in knowledge identified herein. This should include investigations into cat behaviour and physiology with respect to the cat-child and catdog relationship. The cat-owner relationship is additionally worthy of further exploration, given the importance this variable has been to studies so far.

It is additionally important to ensure future results are as meaningful as possible. Standardisation of methodologies such as ethograms and measuring CSS could be beneficial. Whilst results have been relatively consistent thus far with varying methodologies, standardisation would improve the reliability of comparing results between studies. It is also important for studies to ensure sample sizes are sufficient to provide reliable results. Thus far, sample sizes have often been small, even $\mathrm{n}=1$, reducing the ability to apply results to scenarios other than the case being studied.

Complexity is another area which is lacking. Whilst single-variable studies are useful, it is important to understand how variables fit into the bigger picture. Once factors are considered within the environment as a whole, the effect of the variable may be diminished by other environmental factors, and of the majority of indoor cats, environments are not well controlled, but rather dynamic and constantly evolving.

\section{Funding}

This research did not receive any specific grant from funding agencies in the public, commercial or not-for-profit sectors.

\section{Appendix A. Supplementary data}

Supplementary material related to this article can be found, in the online version, at doi:https://doi.org/10.1016/j.applanim.2019. 104841.

\section{References}

Adamelli, S., Marinelli, L., Normando, S., Bono, G., 2005. Owner and cat features influence the quality of life of the cat. Appl. Anim. Behav. Sci. 94 (1-2), 89-98. https:// doi.org/10.1016/j.applanim.2005.02.003.

Amat, M., de la Torre, J.L.R., Fatjó, J., Mariotti, V.M., Van Wijk, S., Manteca, X., 2009. Potential risk factors associated with feline behaviour problems. Appl. Anim. Behav. Sci. 121 (2), 134-139. https://doi.org/10.1016/j.applanim.2009.09.012.

APPA, 2018. The 2017-2018 APPA National Pet Owners Survey Debut. Available at: https://americanpetproducts.org/Uploads/MemServices/GPE2017 NPOS Seminar. pdf (Accessed 17 April 2019).

ASPCA (n.d.) Shelter Intake and Surrender. Pet statistics. Available at: https://www. aspca.org/animal-homelessness/shelter-intake-and-surrender/pet-statistics (Accessed 17 April 2019).

Australian Capital Territory Government, 2018. Transport Canberra and City Services. Cat Containment. Available at: https://www.tccs.act.gov.au/city-living/pets/cats/ cat-containment (Accessed 17 April 2019).

Barry, K.J., Crowell-Davis, S.L., 1999. Gender differences in the social behaviour of the neutered indoor-only domestic cat. Appl. Anim. Behav. Sci. 64 (3), 193-211. https:// doi.org/10.1016/S0168-1591(99)00030-1.

Bernstein, P.L., Strack, M., 1996. A game of cat and house: spatial patterns and behaviour of 14 domestic cats (Felis catus) in the home. Anthrozos 9 (1), 25-39. https://doi. org/10.2752/089279396787001572.

Bradshaw, J., 2018. Normal Feline Behaviour ... and why problem behaviours develop. J. Feline Med. Surg. 20 (5), 411-421. https://doi.org/10.1177/1098612X18771203.

Bradshaw, J.W., Hall, S.L., 1999. Affiliative behaviour of related and unrelated pairs of cats in catteries: a preliminary report. Appl. Anim. Behav. Sci. 63 (3), 251-255. https://doi.org/10.1016/S0168-1591(99)00007-6.

Broadley, H.M., McCobb, E.C., Slater, M.R., 2014. Effect of single-cat versus multi-cat home history on perceived behavioural stress in domestic cats (Felis silvestrus catus) in an animal shelter. J. Feline Med. Surg. 16 (2), 137-143. https://doi.org/10.1177/ $1098612 X 13502972$.

Broom, D.M., 1986. Indicators of poor welfare. Br. Vet. J. 142 (6), 524-526. https://doi. org/10.1016/0007-1935(86)90109-0.

Carlstead, K., Brown, J.L., Strawn, W., 1993. Behavioural and physiological correlates of stress in laboratory cats. Appl. Anim. Behav. Sci. 38 (2), 143-158. https://doi.org/10 1016/0168-1591(93)90062-T.

Casey, R.A., Vandenbussche, S., Bradshaw, J.W.S., Roberts, M.A., 2009. Reasons for relinquishment and return of domestic cats (Felis silvestris catus) to rescue shelters in the UK. Anthrozoos 22 (4), 347-358. https://doi.org/10.2752/ $089279309 X 12538695316185$.

Chadwin, R.M., Bain, M.J., Kass, P.H., 2017. Effect of a synthetic feline facial pheromone product on stress scores and incidence of upper respiratory tract infection in shelter cats. J. Am. Vet. Med. Assoc. 251 (4), 413-420. https://doi.org/10.2460/javma.251. 4.413

Clark, C.C.A., Gruffydd-Jones, T., Murray, J.K., 2012. Number of cats and dogs in UK welfare organisations. Vet. Rec. 170 (19), 493. https://doi.org/10.1136/vr.100524.

Damasceno, J., Genaro, G., 2014. Dynamics of the access of captive domestic cats to a feed environmental enrichment item. Appl. Anim. Behav. Sci. 151, 67-74. https:// doi.org/10.1016/j.applanim.2013.11.004.

Dantas-Divers, L.M.S., Crowell-Davis, S.L., Alford, K., Genaro, G., D’Almeida, J.M., Paixao, R.L., 2011. Agonistic behaviour and environmental enrichment of cats communally housed in a shelter. J. Am. Vet. Med. Assoc. 239 (6), 796-802. https:// doi.org/10.2460/javma.239.6.796.

de Monte, M., le Pape, G., 1997. Behavioural effects of cage enrichment in single-caged adult cats. Anim. Welf. 6 (1), 53-66.

de Oliveira, A.S., Terçariol, C.A.S., Genaro, G., 2015. The use of refuges by communally housed cats. Animals 5 (2), 245-258. https://doi.org/10.3390/ani5020245.

Desforges, E.J., Moesta, A., Farnworth, M.J., 2016. Effect of a shelf-furnished screen on space utilisation and social behaviour of indoor group-housed cats (Felis silvestris catus). Appl. Anim. Behav. Sci. 178, 60-68. https://doi.org/10.1016/J.APPLANIM 2016.03.006.

Driscoll, C.A., Menotti-Raymond, M., Roca, A.L., Hupe, K., Johnson, W.E., Geffen, E. Harley, E.H., Delibes, M., Pontier, D., Kitchener, A.C., Yamaguchi, N., O'Brien, S.J., Macdonald, D.W., 2007. The near eastern origin of cat domestication. Science 317 (5837), 519-523. https://doi.org/10.1126/science.1139518.

Ellis, J.J., McGowan, R.T.S., Martin, F., 2017a. Does previous use affect litter box appeal in multi-cat households? Behav. Processes 141, 284-290. https://doi.org/10.1016/j. beproc.2017.02.008.

Ellis, J.J., Stryhn, H., Spears, J., Cockram, M.S., 2017b. Environmental enrichment choices of shelter cats. Behav. Processes 141, 291-296. https://doi.org/10.1016/j. beproc.2017.03.023.

Ellis, S.L.H., Wells, D.L., 2008. The influence of visual stimulation on the behaviour of cats housed in a rescue shelter. Appl. Anim. Behav. Sci. 113 (1-3), 166-174. https:// doi.org/10.1016/j.applanim.2007.11.002.

Ellis, S.L.H., Thompson, H., Guijarro, C., Zulch, H.E., 2015. The influence of body region, handler familiarity and order of region handled on the domestic cat's response to being stroked. Appl. Anim. Behav. Sci. 173, 60-67. https://doi.org/10.1016/J. applanim.2014.11.002.

Eriksson, M., Keeling, L.J., Rehn, T., 2017. Cats and owners interact more with each other after a longer duration of separation. PLoS One 12 (10). https://doi.org/10.1371/ journal.pone.0185599. e0185599-e0185599.

Fazio, E., Ferlazzo, A., Cravana, C., Medica, P., 2017. Comparison of acute versus chronic stress responses to different housing's systems of cats. Acta Sci. Vet. 45, 1493.

Feuerstein, N., Terkel, J., 2008. Interrelationships of dogs (Canis familiaris) and cats (Felis catus L.) living under the same roof. Appl. Anim. Behav. Sci. 113 (1-3), 150-165. https://doi.org/10.1016/j.applanim.2007.10.010.

Finka, L.R., Ward, J., Farnworth, M.J., Mills, D.S., 2019. Owner personality and the wellbeing of their cats share parallels with the parent-child relationship. PLoS One 14 (2). https://doi.org/10.1371/journal.pone.0211862.

Gouveia, K., Magalhães, A., de Sousa, L., Magalhaes, A., de Sousa, L., 2011. The behaviour of domestic cats in a shelter: residence time, density and sex ratio. Appl. Anim. Behav. Sci. 130 (1-2), 53-59. https://doi.org/10.1016/j.applanim.2010.12.009.

Grigg, E.K., Pick, L., Nibblett, B., 2012. Litter box preference in domestic cats: covered versus uncovered. J. Feline Med. Surg. 15 (4), 280-284. https://doi.org/10.1177/ 1098612 X12465606.

Hart, L.A., Hart, B.L., Thigpen, A.P., Willits, N.H., Lyons, L.A., Hundenski, S., 2018. Compatibility of cats with children in the family. Front. Vet. Sci. 5, 278. https://doi. org/10.3389/fvets.2018.00278.

Heidenberger, E., 1997. Housing conditions and behavioural problems of indoor cats as assessed by their owners. Appl. Anim. Behav. Sci. 52 (3-4), 345-364. https://doi.org/ 10.1016/S0168-1591(96)01134-3.

Heimbürge, S., Kanitz, E., Otten, W., 2019. The use of hair cortisol for the assessment of stress in animals. Gen. Comp. Endocrinol. 270, 10-17. https://doi.org/10.1016/j. ygcen.2018.09.016.

Herron, M.E., Buffington, C.A.T., 2010. Environmental enrichment for indoor cats. Compend. 32 (12)

Van Herwijnen, I.R., van der Borg, J.A.M., Naguib, M., Beerda, B., 2018. Dog ownership 
satisfaction determinants in the owner-dog relationship and the dog's behaviour. PLoS One 13 (9). https://doi.org/10.1371/journal.pone.0204592.

International Cat Care (n.d. a) Indoor vs Outdoor. Advice. Found at: https://icatcare.org/ advice/keeping-your-cat-happy/indoors-versus-outdoors (Accessed 4 April 2019).

International Cat Care (n.d. b) Satisfying the needs of the indoor cat. Advice. Found at: https://icatcare.org/advice/keeping-your-cat-happy/indoors-versus-outdoors (Accessed 4 April 2019).

Kennedy, C.J., Thomson, A.E., Griffith, E.H., Fogle, J., Lascelles, B.D.X., Meeker, R.B., Sherman, B.L., Gruen, M.E., 2018. Enrichment preferences of FIV-infected and uninfected laboratory-housed cats. Viruses 10 (7), 1-10. https://doi.org/10.3390/ v10070353.

Kessler, M.R., Turner, D.C., 1997. Stress and adaptation of cats (Felis silvestris catus) housed singly, in pairs and in groups in boarding catteries. Anthrozoos 4 (4), 214-231.

Kessler, M.R., Turner, D.C., 1999a. Socialization and stress in cats (Felis silvestris catus) housed singly and in groups in animal shelters. Anim. Welf. 8 (1), 15-26.

Kessler, M.R., Turner, D.C., 1999b. Effects of density and cage size on stress in domestic cats (Felis silvestris catus) housed in animal shelters and boarding catteries. Anim. Welf. 8 (3), 259-267.

Kogan, L., Kolus, C., Schoenfeld-Tacher, R., 2017. Assessment of clicker training for shelter cats. Animals 7 (10). https://doi.org/10.3390/ani7100073.

Kry, K., Casey, R., 2007. The effect of hiding enrichment on stress levels and behaviour of domestic cats (Felis silvestris catus) in a shelter setting and the implications for adoption. Anim. Welf. 16, 375-383.

Linklater, W.L., Farnworth, M.J., Van Heezik, Y., Stafford, K.J., Macdonald, E.A., 2019 Prioritising cat-owner behaviours for a campaign to reduce wildlife depredation. Conserv. Sci. Practice Forthcoming.

Lichtsteiner, M., Turner, D., 2008. Influence of indoor-cat group size and dominance rank on urinary cortisol levels. Anim. Welf. 17, 215-237.

Loberg, J.M., Lundmark, F., 2016. The effect of space on behaviour in large groups of domestic cats kept indoors. Appl. Anim. Behav. Sci. 182, 23-29. https://doi.org/10. 1016/j.applanim.2016.05.0300168-1591.

Marlborough District Council (n.d.) Cats. Available at: https://www.marlborough.govt. nz/services/dogs-and-other-animals/cats (Accessed 26 March 2019).

McCobb, E.C., Patronek, G.J., Marder, A., Dinnage, J.D., Stone, M.S., 2005. Assessment of stress levels among cats in four animal shelters. J. Am. Vet. Med. Assoc. 226 (4), 548-555. https://doi.org/10.2460/javma.2005.226.548.

McCune, S., 1994. Caged Cats: Avoiding Problems and Providing Solutions. Newsletter of the Companion Animal Study Group No 7.

McGlone, J.J., Garcia, A., Thompson, W.G., Pirner, G.M., 2018. Maternal-neonatal Pheromone/Interomone added to cat litter improves litter Box use and reduces aggression in pair-housed cats. J. Appl. Anim. Welf. Sci. 1-12. https://doi.org/10.1080/ 10888705.2018.1446341.

McLeod, L.J., Hine, D.W., Bengsen, A.J., 2015. Born to roam? Surveying cat owners in Tasmania, Australia, to identify the drivers and barriers to cat containment. Prev. Vet. Med. 122 (3), 339-344. https://doi.org/10.1016/J.PREVETMED.2015.11.007.

Mertens, C., 1991. Human-cat interactions in the home setting. Anthrozos 4 (4), 214-231. https://doi.org/10.2752/089279391787057062.

Moher, D., Liberati, A., Tetzlaff, J., Altman, D.G., The PRISMA Group, 2009. Preferred Reporting Items for Systematic Reviews and Meta-Analyses: The PRISMA Statement. PLoS Med. 6 (7). https://doi.org/10.1371/journal.pmed1000097.

Moore, A.M., Bain, M.J., 2013. Evaluation of the addition of in-cage hiding structures and toys and timing of administration of behavioural assessments with newly relin quished shelter cats. J. Vet. Behav.: Clin. Appl. Res. 8 (6), 450-457. https://doi.org/ 10.1016/j.jveb.2011.10.003.

Murray, J.K., Roberts, M.A., Whitmarsh, A., Gruffydd-Jones, T.J., 2009. Survey of the characteristics of cats owned by households in the UK and factors affecting their neutered status. Vet. Rec. 164 (5), 137-141. https://doi.org/10.1136/vr.164.5.137.

Naik, R., Witzel, A., Albright, J.D., Siegfried, K., Gruen, M.E., Thomson, A., Price, J., Lascelles, B.D.X., 2018. Pilot study evaluating the effect of feeding method on overall activity of neutered indoor pet cats. J. Vet. Behav. 25, 9-13. https://doi.org/10. 1016/j.jveb.2018.02.001.

Ottway, D.S., Hawkins, D.M., 2003. Cat housing in rescue shelters: a welfare comparison between communal and discrete-unit housing. Anim. Welf. 12 (2), 173-189.

Parker, M., Lamoureux, S., Allouche, B., Brossier, J.-A., Weber, M., Feugier, A., Moniot, D., Deputte, B., Biourge, V., Serra, J., 2017. Accuracy assessment of spatial organization and activity of indoor cats using a system based on ultrawide band technology. J. Vet. Behav.: Clin. Appl. Res. 21, 13-19. https://doi.org/10.1016/J.JVEB.2017.06. 003.

Patronek, G.J., Glickman, L.T., Beck, A.M., McCabe, G.P., Ecker, C., 1996. Risk factors for relinquishment of cats to an animal shelter. J. Am. Vet. Med. Assoc. 209 (3), 582-588.

PDSA, 2017. Issues in focus. PAW Report 2017. Available at: https://www.pdsa.org.uk/ get-involved/our-current-campaigns/pdsa-animal-wellbeing-report (Accessed 13 September 2018).

Podberscek, A., Blackshaw, J.K., Beattie, A.W., 1991. The behaviour of laboratory colony cats and their reactions to a familiar and unfamiliar person. Appl. Anim. Behav. Sci. 31, 119-130.

Ramos, D., Reche-Junior, A., Fragoso, P.L.L., Palme, R., Yanasse, N.K.K., Gouvêa, V.R.R., Beck, A., Mills, D.S.S., 2013. Are cats (Felis catus) from multi-cat households more stressed? Evidence from assessment of faecal glucocorticoid metabolite analysis. Physiol. Behav. 122, 72-75. https://doi.org/10.1016/j.physbeh.2013.08.028.

Ramos, D., Arena, M.N., Reche Jr, A., Daniel, A.G.T., Albino, M.V.C., Vasconcellos, A.S., Viau, P., Oliveira, C.A., 2012. Factors affecting faecal glucocorticoid levels in domestic cats (Felis catus): a pilot study with single and large multi-cat households. Anim. Welf. 21 (2), 285-291. https://doi.org/10.7120/09627286.21.2.285.

Rehnberg, L.K., Robert, K.A., Watson, S.J., Peters, R.A., 2015. The effects of social interaction and environmental enrichment on the space use, behaviour and stress of owned housecats facing a novel environment. Appl. Anim. Behav. Sci. 169, 51-61. https://doi.org/10.1016/j.applanim.2015.06.002.

Rochlitz, I., Podberscek, A.L., Broom, D.M., 1998a. Welfare of cats in a quarantine cattery. Vet. Rec. 143 (2), 35-39. https://doi.org/10.1136/vr.143.2.35.

Rochlitz, I., Podberscek, A.L., Broom, D.M., 1998b. Effects of quarantine on cats and their owners. Vet. Rec. 143 (7), 181-185. https://doi.org/10.1136/vr.143.7.181.

Sandøe, P., Nørspang, A.P., Forkman, B., Bjørnvad, C.R., Kondrup, S.V.S., Lund, T.B., 2017. The burden of domestication: a representative study of welfare in privately owned cats in Denmark. Anim. Welf. 26 (1), 1-10. https://doi.org/10.7120/ 09627286.26.1.001.

Schubnel, E., Arpaillange, C., 2008. Contribution to the study of indoor cats' behavioural problems. Pratique Medicale et Chirurgicale de l'Animal de Compagnie 43 (2), 63-70. https://doi.org/10.1016/j.anicom.2008.05.001.

Shyan-Norwalt, M.R., 2005. Caregiver perceptions of what indoor cats do "For fun". J. Appl. Anim. Welf. Sci. 8 (3), 199-209. https://doi.org/10.1207/ s15327604jaws0803_4.

Snowdon, C.T., Teie, D., Savage, M., 2015. Cats prefer species-appropriate music. Appl. Anim. Behav. Sci. 166, 106-111. https://doi.org/10.1016/j applanim.2015.02.012.

Soennichsen, S., Chamove, A.S., 2002. Responses of cats to petting by humans. Anthrozos 15 (3), 258-265. https://doi.org/10.2752/089279302786992577.

Stavisky, J., Brennan, M.L., Downes, M., Dean, R., 2012. Demographics and economic burden of un-owned cats and dogs in the UK: results of a 2010 census. BMC Vet. Res. 8 (1), 163. https://doi.org/10.1186/1746-6148-8-163.

Stella, J., Croney, C., Buffington, T., 2014. Environmental factors that affect the behaviour and welfare of domestic cats (Felis silvestris catus) housed in cages. Appl. Anim. Behav. Sci. 160 (1), 94-105. https://doi.org/10.1016/j.applanim.2014.08.006.

Stella, J., Croney, C., Buffington, T., 2017. Behaviour and welfare of domestic cats housed in cages larger than US norm. J. Appl. Anim. Welf. Sci. 20 (3), 296-312. https://doi. org $/ 10.1080 / 10888705.2017 .1317252$.

Strickler, B.L., Shull, E.A., 2014. An owner survey of toys, activities, and behaviour problems in indoor cats. Journal of Veterinary Behaviour: Clinical Applications and Research 9 (5), 207-214. https://doi.org/10.1016/j.jveb.2014.06.005.

Taborsky, M., 2010. Sample size in the study of behaviour. Ethology 116 (3), 185-202. https://doi.org/10.1111/j.1439-0310.2010.01751.x.

Thomson, J.E., Hall, S.S., Mills, D.S., 2018. Evaluation of the relationship between cats and dogs living in the same home. J. Vet. Behav. 27, 35-40. https://doi.org/10.1016/ J.JVEB.2018.06.043.

Toukhsati, S.R., Young, E., Bennett, P.C., Coleman, G.J., 2012. Wandering cats: attitudes and behaviours towards cat containment in Australia. Anthrozos 25 (1), 61-74.

Uetake, K., Goto, A., Koyama, R., Kikuchi, R., Tanaka, T., 2013. Effects of single caging and cage size on behaviour and stress level of domestic neutered cats housed in an animal shelter. Anim. Sci. J. 84 (3), 272-274. https://doi.org/10.1111/j.1740-0929. 2012.01055.x.

van den Bos, R., 1998a. The function of allogrooming in domestic cats (Felis silvestris catus); a study in a group of cats living in confinement. J. Ethol. 16 (1), 1-13. https:// doi.org/10.1007/BF02896348.

van den Bos, R., 1998b. Post-conflict stress-response in confined group-living cats (Felis silvestris catus). Appl. Anim. Behav. Sci. 59 (4), 323-330. https://doi.org/10.1016/ S0168-1591(98)00147-6.

van den Bos, R., Buning, TdeC., 1994. Social behaviour of domestic cats (Felis lybica f. Catus L.): study of dominance in a group of female laboratory cats. Ethology 98 (1), 14-37. https://doi.org/10.1111/j.1439-0310.1994.tb01054.x.

Vinke, C.M., Godijn, L.M., van der Leij, W.J.R., 2014. Will a hiding box provide stress reduction for shelter cats? Appl. Anim. Behav. Sci. 160 (1), 86-93. https://doi.org/ 10.1016/j.applanim.2014.09.002.

Vitale Shreve, K.R., Mehrkam, L.R., Udell, M.A.R., 2017. Social interaction, food, scent or toys? A formal assessment of domestic pet and shelter cat (Felis silvestris catus) preferences. Behav. Processes 141, 322-328. https://doi.org/10.1016/j.beproc.2017. 03.016.

Wedl, M., Bauer, B., Gracey, D., Grabmayer, C., 2011. Factors Influencing the Temporal Patterns of Dyadic Behaviours and Interactions between Domestic Cats and Their Owners 86. Elsevier, pp. 58-67. https://doi.org/10.1016/j.beproc.2010.09.001.

Zhang, L., Plummer, R., McGlone, J., 2018. Preference of kittens for scratchers. J. Feline Med. Surg. https://doi.org/10.1177/1098612X18795258. 